\title{
A TURBIDIMETRIC METHOD FOR THE ASSAY OF ANTIBIOTICS
}

\author{
DWIGHT A. JOSLYN AND MARGARET GALBRAITH \\ Research Laboratories, Parke, Davis \& Company, Detroit S2, Michigan
}

Received for publication February 17, 1950

Turbidimetric assay procedures for antibiotic substances have been proposed by Foster (1942); McMahan (1944); Lee, Foley, and Epstein (1944); and Joslyn (1944). Standard methods for the assay of penicillin and streptomycin are used by the Food and Drug Administration (Federal Register, 1947, and recent revisions) and by manufacturers of these antibiotics. Not one of these procedures is exactly applicable to the assay of antibiotic materials for which no standards have been established.

\section{EXPERIMENTAL DEVELOPMENT}

In order to develop a method that would be suitable for determining the antibiotic strength of experimental preparations, such as broth filtrates and chemical fractions as well as the finally purified substance, growth curves of many pathogenic bacteria were studied under varying conditions. Variables that were investigated include the amount of inoculum, the type of nutriment, and the presence or absence of antibacterial substances. The effect of varying the size of the inoculum is shown in figure 1. Figure 2 illustrates the differences between three different nutrients. The effect of the presence of small amounts of an antibacterial agent may be seen in figure 3 .

One characteristic of these data is that, after a period of approximately 4 hours, turbidity, which is indicative of active growth, begins to level off. This is interpreted as signifying that the period of most active growth occurs before the cultures are 4 hours old. In order to demonstrate this more conclusively and to point out any significant secondary increase in growth during a 24-hour period, a series of cultures were studied by ascertaining their growth according to turbidimetric readings over a 26 -hour period. Figure 4 presents typical results of this portion of the study. These curves demonstrate that the period of most active growth in each case occurred during the first 4-hour period.

Examination of growth curves altered by the presence of antibacterial agents shows that there are apparently two different types of modification. In one, the growth curves of cultures containing increasing amounts of antibiotic fan out in a fairly regular manner. Each curve that contains a greater portion of antibiotic agent departs further from the "normal" in a regular and definite manner. In the other, the fanning out is less orderly, and not until the amount of antibacterial substance is relatively large does the growth curve depart markedly from the "normal" and growth of the culture suddenly become nearly negligible. In the latter instance there is a great difference in the behavior of growth curves of cultures of bacteria containing only slightly different concentrations of antibacterial substance. 

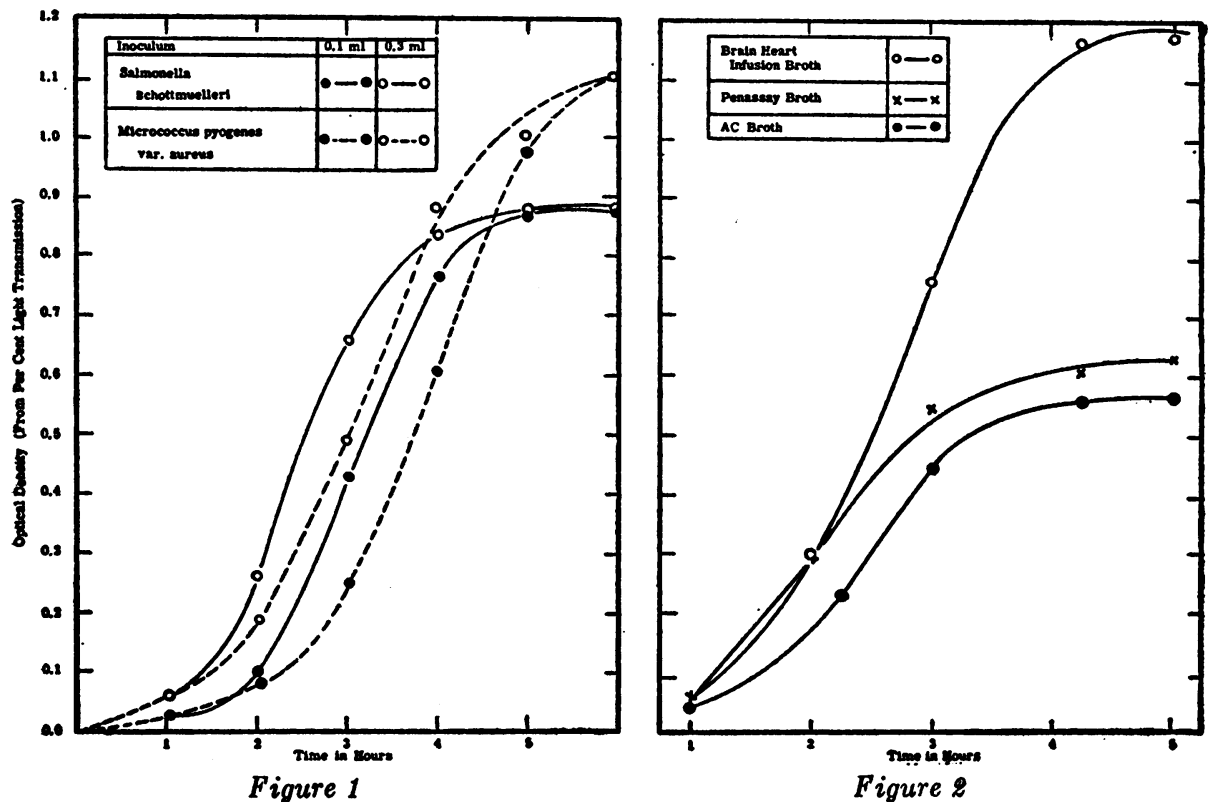

Figure 1. Effect of inoculum size on the growth curves of two species. Figure 8. Growth curves of Shigella sonnei in three Difco media.
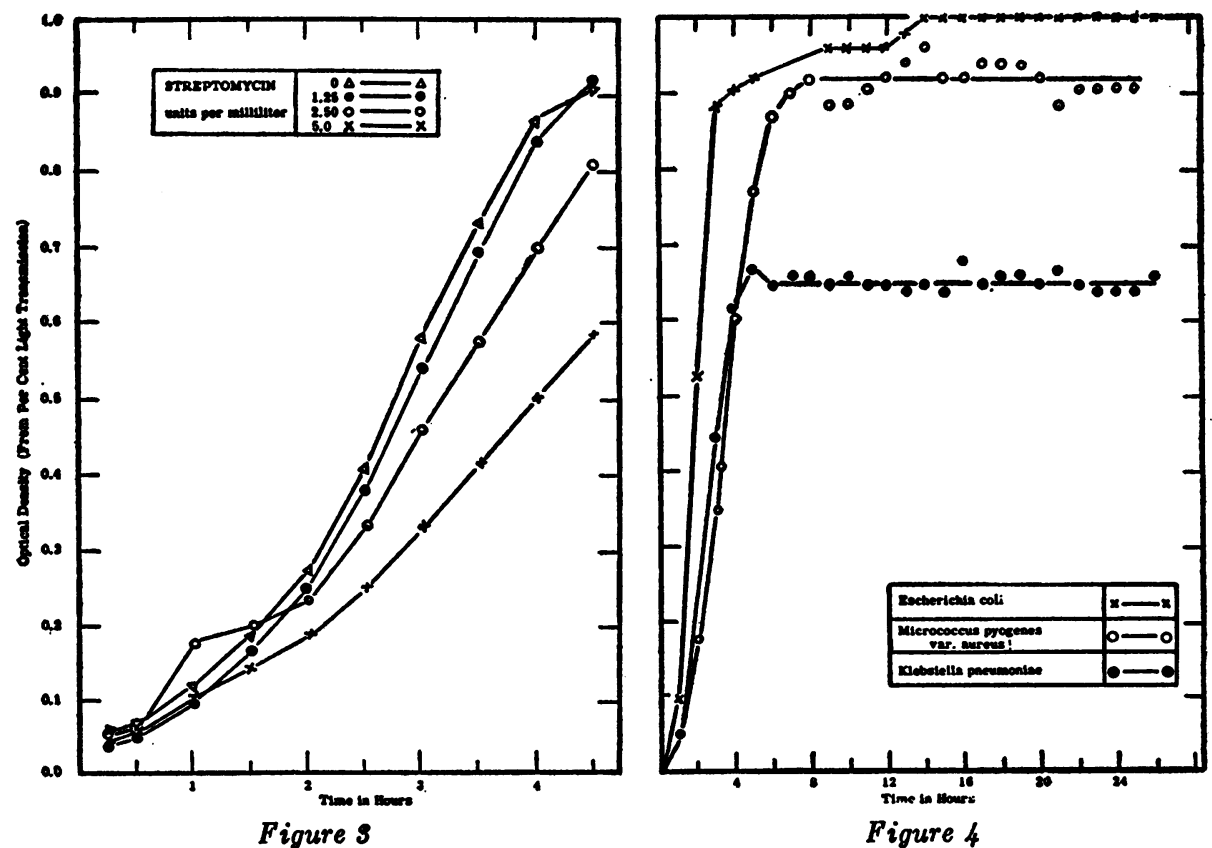

Figure 3. Effect of antibiotic concentration on growth of Micrococcus pyogenes var. aureus. Figure 4. Growth curves showing early attainment of maximum turbidity.

Another occurrence that attracted attention was the fact that the lag phase of cultures could almost be eliminated if a sizable inoculum of a very young 
culture was employed. That is, if 0.4 to $0.8 \mathrm{ml}$, instead of the usual $0.2 \mathrm{ml}$, of inoculum of a 2- to 4-hour culture is added to broth to make a total volume of $10 \mathrm{ml}$, the lag phase becomes negligible. However, the best inoculum for the present test must be considered to be the smallest amount of susceptible culture that can be employed and that will still reach the desired percentage of light transmission within 3 to 4 hours. This amount must also be sensitive to a very small quantity of the antibiotic to be assayed. The smaller the inoculum, the greater is the effect of a given concentration of the antibiotic substance.

Upon consideration of these facts, it was reasoned that alteration of the growth curve would be a convenient as well as an accurate method of determining the antibiotic potentialities of metabolic products, and it was decided that the period of most active growth would be the most useful in developing a sensitive method of assay. Hence the inoculum for the assay was taken from the most active growing period and the test terminated before the "leveling off" period of the growth curve.

The actual method of measuring the alteration of a growth curve was not originally apparent. Among other methods, measurement of the angle between the normal and altered growth curves, estimation of the distance between the normal and altered curve at a certain point from the base, and measurement of the area under the two curves were considered. It was finally decided that a culture control would be employed; and before this control reached its maximum growth the test would be terminated, and the end point would be the amount of an antibiotic that inhibits the growth of the test organism by 50 per cent. Accordingly the following outline evolved as a method for determining the potency of antibiotic agents. This method has already been described briefly (Joslyn and Galbraith, 1947; Smith et al., 1948); it is here presented in greater detail.

\section{METHOD USED IN THE ABSENCE OF A STANDARD}

Because of the heavy inoculum and short incubation period employed, samples need not be sterilized, unless heavily contaminated, provided that ordinary aseptic precautions are taken. A series of dilutions of the unknown, based upon estimated potency, are made, and 1-ml amounts of each dilution are placed in a series of matched test tubes. ${ }^{1}$ In the last tube of the series to which no antibiotic has been added $1.0 \mathrm{ml}$ sterile distilled water is placed. The last tube serves as the "culture control" and is an integral part of each row of test tubes that make up the test. Next is added $8.8 \mathrm{ml}$ of 1.1-strength brain heart infusion (Difco) broth. Inoculation is effected by adding $0.2 \mathrm{ml}$ of culture, held in an ice bath after reaching an optical density of 0.456 (35 per cent light transmission on the "lumetron"). (For example, 0.2 to $0.4 \mathrm{ml}$ of Escherichia coli, previously prepared by being grown at $37 \mathrm{C}$ for 5 hours, is transferred to fresh broth, grown at 37 $C$ until the optical density reaches 0.456 - usually about 2 hours- and diluted 1:8.) As each row, including its culture control, is inoculated, it is placed in a $37 \mathrm{C}$ water bath, where it remains until the light transmission of the culture con-

1 Test tubes $(18 \times 150 \mathrm{~mm})$ are matched to read equally in the photoelectric colorimeter ("lumetron," model 402E). 
trol reaches 38 per cent (optical density 0.420$).{ }^{2}$ At this point the rows are successively removed, wiped dry, and shaken, and the light transmissions are recorded after the colorimeter ${ }^{3}$ is set by using an uninoculated and usually unincubated ${ }^{4}$ broth tube at 100 per cent light transmission. From a convenient table the optical density for each light transmission is recorded. The percentage of growth of each dilution is calculated by dividing the optical density of the test dilution by the optical density of the control and multiplying by 100 . The percentage of inhibition is determined by subtraction of the percentage of growth from 100. Fifty per cent inhibition is used as the end point. No statistical analysis of this method has been attempted, but replicate assays upon successive days usually agree within 5 per cent.

This method has been found suitable for a number of bacteria. Organisms that we have used successfully are Aerobacter aerogenes, Bacillus mycoides, Escherichia coli, Klebsiella pneumoniae, Micrococcus pyogenes var. aureus, Pasteurella bollingeri, Proteus sp., Salmonella schottmuelleri, Salmonella typhosa, Serratia marcescens, Shigella sonnei, and several Streptococci. Inocula for all but three of these cultures are used undiluted when their optical density reaches 0.456 (35 per cent light transmission). Inocula for $E$. coli, S. schottmuelleri, and S. sonnei are diluted $1: 8$.

METHOD USED IN THE PRESENCE OF A STANDARD: CHLORAMPHENICOL ASSAY

This method is suitable for the assay of any antibiotic for which a reference standard is available. In this instance the procedure is exactly the same except that duplicate rows of the standard are run in the same manner as dilutions of the unknown.

The assay of chloramphenicol is begun by accurately weighing a sample of the standard and making a solution containing $50 \mu \mathrm{g}$ per $\mathrm{ml}$ with sterile distilled water. This standard solution may be kept at refrigerator temperature for at least a month without loss in activity. Dilutions of this standard are made to represent 1 in 10,15,20,30, and 40. These correspond to final dilutions in the test tubes of 1 in 100,150,200,300, and 400. One-ml amounts of each of these dilutions are placed in separate tubes of a duplicate series of five matched test tubes. In a sixth similar tube for each row of both standard and unknown is placed $1.0 \mathrm{ml}$ of sterile distilled water. These are employed as culture controls. Similar dilutions of the unknown samples, based upon estimated potency, are made, and 1.0-ml amounts are pipetted to matched tubes. Next is added to each

2 $L=2-\log G$ where $L=$ density and $G=$ galvanometer reading (percentage of transmission).

"The "lumetron," model 402E, is the colorimeter used in this method. However, the "junior spectrophotometer" (Coleman) may be used instead of the "lumetron." A light transmission of 35 per cent on the "lumetron" using a 575-m $\mu$ filter is equivalent to 70 on the "Coleman" set at a wavelength of 575 millimicrons.

- When serum samples are tested, the color control tubes are incubated for the length of the test because there is a change in color upon incubation. Some color controls for chemical compounds have also been incubated because some chemicals precipitate the medium when incubated at $37 \mathrm{C}$ for several hours. 


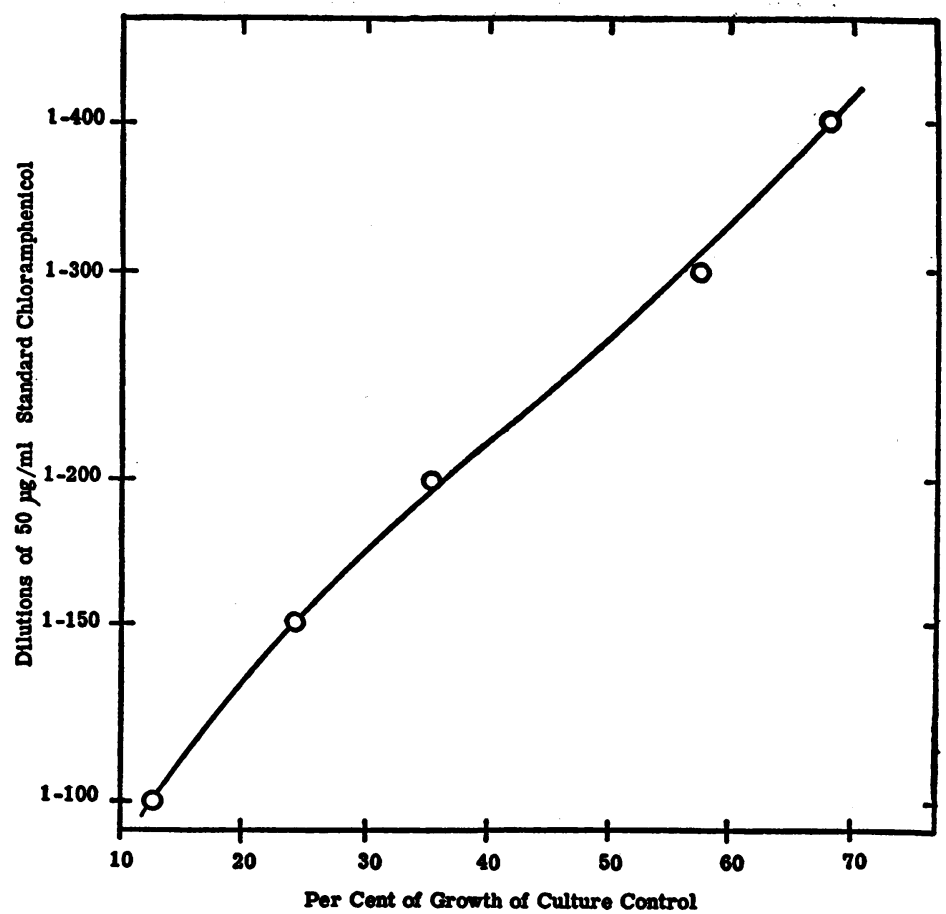

Figure 5. Typical daily chloramphenicol standard curve of Shigella sonnei.

TABLE I

Chloramphenicol assay: calculation of potency

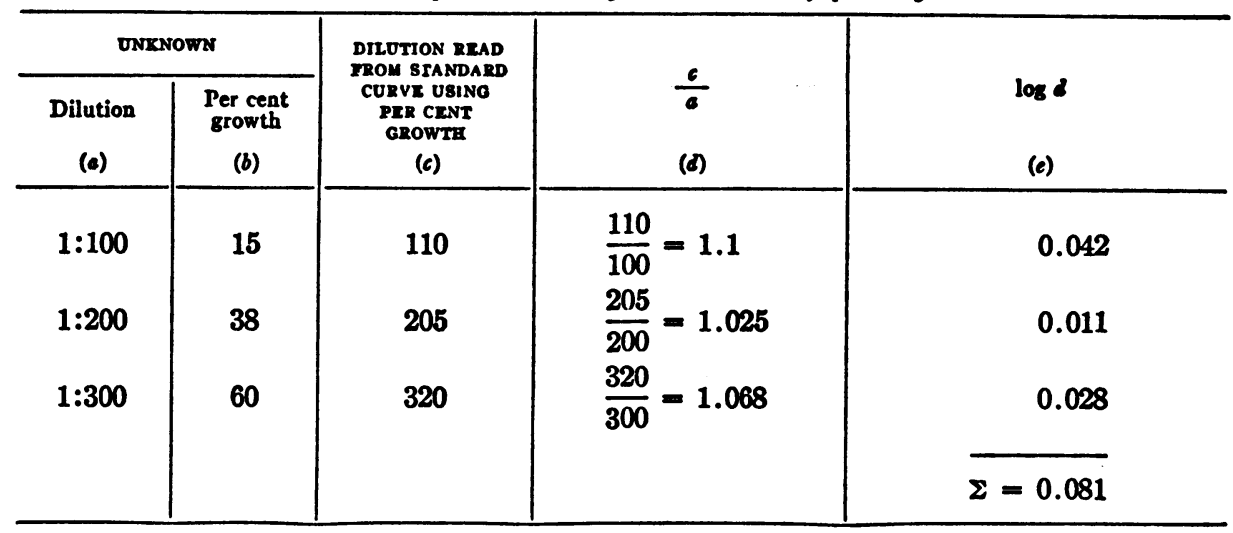

$\frac{\Sigma e=0.081}{3}=0.027$ (logarithmic mean)

$\log 50-0.027=1.672$

Antilog $1.672=47 \mu \mathrm{g}$ per $\mathrm{ml}=$ concentration of unknown

As may be seen from this example, the percentage of growth (b) of each dilution (a) of the unknown is translated by means of the standard curve into terms of the corresponding dilution of the standard $(c)$. The value thus obtained is divided by the actual dilution of the unknown $(d)$. The logarithmic mean of these values $(e)$ is obtained and subtracted from the log of 50 (because $50 \mu \mathrm{g}$ per $\mathrm{ml}$ is the concentration of the standard solution and the dilutions plotted for the standard curves are dilutions of this solution). The antilog of this difference is the estimated concentration of the unknown in $\mu \mathrm{g}$ per ml. 
tube $8.8 \mathrm{ml}$ of 1.1-strength brain heart infusion (Difco) broth. Inoculation is effected by adding $0.2 \mathrm{ml}$ of culture (Shigella sonnei) to each tube. The inoculum is prepared by transferring the organisms from a slant culture to broth and growing them at $37 \mathrm{C}$ for 5 hours, then transferring $0.4 \mathrm{ml}$ of the resulting broth culture to $10 \mathrm{ml}$ fresh broth, growing it at $37 \mathrm{C}$ until the optical density reaches 0.456 (usually about 2 hours), and making a 1:8 dilution of it. As each row, including its culture control, is inoculated, it is placed in the $37 \mathrm{C}$ water bath, where it remains until the light transmission of its culture control reaches 38 per cent (density $=0.420 ; 69$ to 72 per cent on the Coleman). At this time the rows are successively removed, wiped dry, and shaken, and the light transmissions are recorded after the colorimeter is set at 100 with an uninoculated broth tube. From a convenient table the optical density for each transmission is recorded. The percentage of growth of each dilution of the standard and unknown is calculated by dividing the optical density of the test dilution by the optical density of the control and multiplying by 100 . The average percentage of growth values for the pairs of standard tubes at each dilution is plotted on a linear scale against the dilutions on a logarithmic scale, and a smooth curve is drawn to connect the points (figure 5). This is the standard curve for all unknowns being assayed concurrently. Potency calculation of the unknown is best visualized by inspection of the example in table 1 .

A variety of samples have been tested for chloramphenicol by means of this assay method. These include (1) production process samples and manufactured products such as capsules, solutions, creams, ointments, and suppositories; and (2) clinical specimens such as serum, spinal fluid, bile, urine, ${ }^{b}$ and tissue juices.

\section{SUMMARY}

A convenient method is described for the determination of antibiotic potencies of crude beers, fractions, and crystalline end products of an antibiotic whether or not a standard has been established.

\section{REFERENCES}

Foster, J. W. 1942 Quantitative estimation of penicillin. J. Biol. Chem., 144, 285-286. JosLYN, D. A. 1944 Penicillin assay: outline of four-hour turbidimetric method. Science, 99, 21-22.

Joslyn, D. A., and Galbratth, M. 1947 A turbidimetric method for the assay of antibiotics. J. Bact., 54, 26.

LER, S. W., Folex, E. J., AND EPsTeIN, J. 1944 Improvements in the turbidimetric assay for penicillin. J. Biol. Chem., 152, 485-486.

McMahan, J. R. 1944 An improved short time turbidimetric assay for penicillin. J. Biol. Chem., 153, 249-258.

Smith, R. M., Joslyn, D. A., Gruhzit, O., McLean, I. W., Jr., Penner, M., and Ehruch, J. 1948 Chloromycetin: biological studies. J. Bact., 65, 425-448.

\footnotetext{
seavily contaminated bile and urine samples are usually sterilized by Seitz filtration.
} 